\title{
Emotion clustering from stimulated electroencephalographic signals using a Duffing oscillator
}

\author{
P. Bhowmik, S. Das and A. Konar* \\ Department of Electronics and Tele-Communication Engineering, \\ Jadavpur University, \\ Calcutta-32, India \\ E-mail: bpave188@gmail.com \\ E-mail: sauvik.095@gmail.com \\ E-mail: konaramit@yahoo.co.in \\ *Corresponding author

\section{Nandi}

Calcutta Institute of Engineering and Management, Calcutta-24, India

E-mail: dolphia85@gmail.com

\author{
A. Chakraborty \\ Department of Computer Science and Engineering, \\ St. Thomas' College of Engineering and Technology, \\ Calcutta-23, India \\ E-mail: aruna_stcet@rediffmail.com
}

\begin{abstract}
The paper proposes an alternative approach to emotion recognition from stimulated EEG signals using Duffing oscillator. Reported works on emotion clustering generally employ the principles of supervised learning. Unfortunately, because of noisy and limited feature set, the classification problem often suffers from high inaccuracy. This has been overcome in this paper by submitting the EEG signals directly to a Duffing oscillator and the phase portraits constructed from its time-response demonstrate structural similarity to similar emotion excitatory stimuli. The accuracy in clustering was experimentally validated even with injection of Gaussian noise over the EEG signal up to a signal-to-noise ratio of $25 \mathrm{~dB}$. The results of clustering in presence of low signal-to-noise ratio confirm the robustness of the proposed scheme.
\end{abstract}

Keywords: Duffing oscillator; EEG; emotion clustering; Gaussian noise.

Reference to this paper should be made as follows: Bhowmik, P., Das, S., Konar, A., Nandi, D. and Chakraborty, A. (2010) 'Emotion clustering from stimulated electroencephalographic signals using a Duffing oscillator', Int. J. Computers in Healthcare, Vol. 1, No. 1, pp.66-85. 
Biographical notes: Pavel Bhowmik currently a final year BE student of Electronics and Telecommunication Engineering Department, Jadavpur University, Kolkata-700032, India. His principal research interests lie in artificial intelligence, especially pattern recognition, cognitive science and human-computer-interaction. Currently, he is working under Prof. Amit Konar. Solving puzzles, collecting stamps and watching movies at leisure hours are some of his hobbies.

Sauvik Das is currently a final year BE student of Electronics and Telecommunication Engineering Department, Jadavpur University, Kolkata-700032, India. His principal research interests lie in artificial intelligence, especially pattern recognition, cognitive science and human-computer-interaction. Currently, he is working under Prof. Amit Konar. In his leisure hours, he likes to listen to music or watch films. He also likes to solve puzzles and riddles.

Amit Konar obtained his BE degree from Bengal Engineering and Science University, Howrah, India, in 1983 and the METelE, MPhil, and PhD (Engineering) from Jadavpur University, in 1985, 1988, and 1994, respectively. $\mathrm{He}$ is a Professor in the Electronics and Tele-communication Engineering Department, Jadavpur University. He has around 200 publications in international journal and conference proceedings. He is the author of seven books. His research areas include the study of computational intelligence algorithms and their applications. He received the All India Council for Technical Education-accredited 1997-2000 Career Award for Young Teachers.

Dolphia Nandi completed her schooling from BE College Model School, securing 72nd rank in 10th Standard and 183rd rank in 12th Standard. She joined Calcutta Institute of Engineering and Management and passed Bachelors of Technology with 8.33 DGPA. At present, she is working under Prof. Amit Konar. Her hobbies are salsa dancing and painting. Her principal research interests are image processing specially pattern recognition. Signal processing and machine learning are two areas she likes to investigate on. In addition, she wants to learn more about artificial intelligence.

Aruna Chakraborty obtained her MA in Cognitive Science and her $\mathrm{PhD}$ in Emotional Intelligence and Human-Computer Interactions from Jadavpur University, Calcutta, India, in 2000 and 2005, respectively. She is an Associate Professor with the Department of Computer Science and Engineering, St. Thomas' College of Engineering and Technology, Calcutta. She is also a Visiting Faculty with Jadavpur University. She, with A. Konar, has written a book Emotional Intelligence: A Cybernetic Approach, which has appeared from Springer, Heidelberg, 2009. Her current research interest includes artificial intelligence, emotion modelling, and their applications in next-generation human-machine interactive systems.

\section{Introduction}

Perception involves interpreting sights, sounds, smells and touch. Perception is a relatively younger discipline in artificial intelligence. We are concerned that there are fewer works on the perception of emotions. Researchers are keen to develop new models 
and techniques in order to understand and recognise emotions resulting from external manifestations, including crying, laughing for example. This paper deals with the classification of emotion aroused by audio-visual stimulus from electroencephalographic (EEGs) signals. Biologists believe that most of our high level understanding processes involving emotions is due to the interaction of neural and hormonal activities. EEGs (Bos, 2007) representing neural activities of the brain may help develop a better understanding of human emotions other than widely used modes, which include facial expression (Ekman and Friesen, 1975; Russell et al., 2002; Yoshitomi et al., 2000) and voice (Russell et al., 2002; Yoshitomi et al., 2000).

In recent times, researchers are taking interest to brain scanning techniques for correctly acquiring relevant information for emotion recognition. The techniques include EEG, magnetoencephalography (MEG) (Dolan et al., 2006), functional magnetic resonance imaging (FMRI) (Johnstone et al., 2006; Schneider et al., 1997; Peyk et al., 2008) and positron emission tomography (PET) (Schneider et al., 1997). Unfortunately, however, little brain functioning could be traced until now using the above imaging techniques. Naturally, very few interesting results have been reported as yet on emotion clustering using the above modes of information extraction. The primary objective of this paper is to classify the emotion of a subject from his or her EEG signal, obtained by audio-visual excitation of the subject. In our original research (Chakroborty, 2005; Chakroborty and Konar, 2009), we classified the input stimulus based on their power to excite a specific emotion. We have again used these stimuli in the present experiment. We like to examine whether the stimuli used for excitation of the same emotion would ultimately map the EEG's onto a unique pattern.

Reported works (Murugappan et al., 2008; Khalili and Moradi, 2008, 2009; Konar and Jain, 2005; Li et al., 2009) on emotion recognition from stimulated EEG signals usually employ supervised learning. Naturally, the classification accuracy of emotions largely depends on the selected features of the EEG signals. As features are usually not free from measurement noise, and the list of complete features is yet to be identified, the classification accuracy sometimes go far below than the other mode of emotion classification. Naturally, the importance of emotion recognition from EEG features becomes insignificant. To overcome this problem, we here use the entire EEG signal samples rather than the features extracted out of it. Here, a non-linear mapping from the stimulated EEG signals to emotion has been accomplished using a relaxation oscillator. There exist several non-linear relaxation oscillators, such as Lee oscillator (Chakroborty and Konar, 2009) and Duffing oscillator (Holmes and Rand, 1976; Holmes and Holmes, 1981; Holmes and Whitley, 1983; Novak and Frehlich, 1982; Srebro, 1995; Ueda, 1979, 1980; Yuan and Li, 2009). We here prefer Duffing oscillator for its proven performance in detecting epileptic seizure from the EEG signals (Yuan and Li, 2009). The novelty of this research is to study the power of Duffing oscillator in emotion clustering from the stimulated EEG signals.

In order to examine the scope of Duffing oscillator in emotion recognition from EEG signals, we consider phase portraits of the oscillator excited with specific audio-visual stimulus responsible for arousal of a given emotion. Experiment reveals that the phase portraits of the oscillator look geometrically similar, when the stimuli correspond to a specific emotion. The paper also examines the clustering of emotions from noisy EEG signals. The classification accuracy obtained by the proposed technique supersedes the accuracy obtained by all other modes of emotion recognition, including facial expression 
(Ekman and Friesen, 1975; Russell et al., 2002; Yoshitomi et al., 2000), voice (Russell et al., 2002; Yoshitomi et al., 2000) and other biopotential signals (Takahashi, 2004).

The paper is divided into five sections. In Section 2, we briefly outline the state space representation of a Duffing oscillator dynamics and how phase trajectories were obtained from the time-response of the oscillator dynamics. In Section 3, we represent the experimental results for emotion clustering by noting the similarity in phase trajectory. The effect of noise on the EEG signal is studied in Section 4. Conclusions derived from the above are listed in Section 5.

\section{Duffing oscillator dynamics and phase response}

Here, we propose a specialised non-linear oscillator dynamics. This has a proven chaotic behaviour (Carroll and Pecora, 1993; Ditto et al., 1990; Novak and Frehlich, 1982) in its temporal response. The dynamics of the Duffing oscillator has a similarity to the typical spring-mass load system of a conventional mechanical process (Kuo, 2002; Ogata, 2009). The spring in the present context, is a non-linear device. It has a restoration force proportional to its cubic linear displacement. Naturally, the restoration force of ideal spring that obeys Hooke's law is maintained in the Duffing oscillator dynamics. Consequently, the restoration force has two components, one following Hooke's law and the other is due to a high stiffness of the spring. This is represented by a cubic displacement term. The dynamics of Duffing oscillator are given in equation (1):

$$
\frac{d^{2} x}{d t^{2}}+\delta \frac{d x}{d t}+\beta x+a x^{3}=\gamma \cos (\omega t)+e(t)
$$

where

$x \quad$ represents the linear displacement

$\frac{d x}{d t}$ represents the velocity of a unit mass connected in spring-mass load system

$\beta x$ and $\alpha x^{3}$ are due to the spring restoration force

$\gamma \cos (\omega t)$ is a fixed excitation input to maintain certain level of oscillation in the response of the dynamics

$e(t)$ is the disturbance input to the oscillator.

In this context, we use the EEG signal as the disturbance input $e(t)$. We select $\alpha=1$, $\beta=-1, \gamma=0.826, \delta=0.5$. The gain of the EEG signal is taken to be 5 . The basic Duffing oscillator dynamics (1) can be represented by (2) and (3).

$$
\begin{aligned}
& \dot{x}=y \\
& \dot{y}=\gamma \cos (\omega t)+e(t)-\delta \frac{d x}{d t}-\beta x+a x^{3}
\end{aligned}
$$

Originally, the EEG signal, obtained in the sampled version, was passed through a first-order-hold circuit, whose transfer function is given by: 


$$
G_{h}(s)=\left[\frac{1+T s}{T}\right] \times\left[\frac{1-e^{-T s}}{s}\right]^{2}
$$

where

\section{$T$ sampling time}

$S \quad$ Laplace-domain operator.

The hold-circuit is used to get a continuous version of the discrete EEG signal. Figure 1 presents a recording scheme of EEG signals. Two computers are used in the said system: one as a stimulator, and the other for recording and analysis of EEG signal data.

Figure 1 EEG recording of emotion joy from an audio-visual stimulus (see online version for colours)

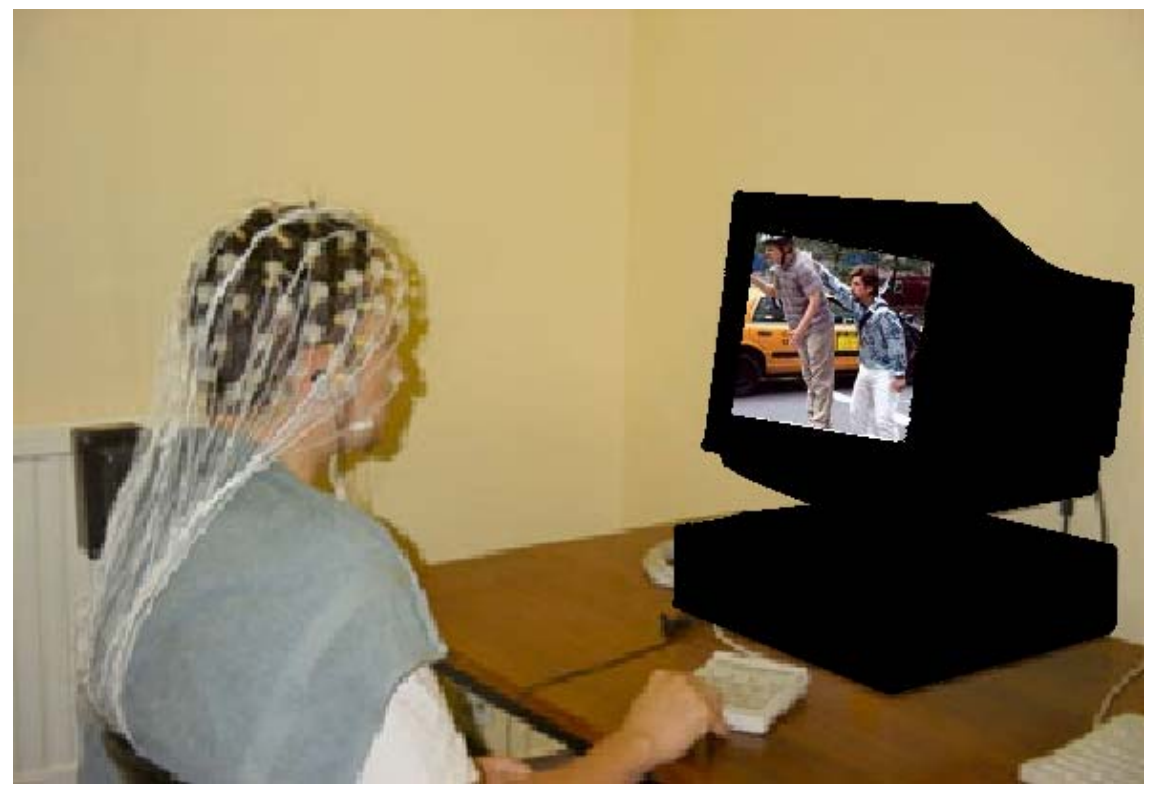

The dynamics of the Duffing oscillator (Figure 2) receives a co-sinusoid input along with the recorded EEG signal. The time-response of the dynamics is obtained numerically by fourth order Runge-Kutta method and phase trajectories are constructed with its parameter $x(t)$ and $\dot{x}(t)$.

Figure 2 Block diagram of a Duffing oscillator

$$
\left.\begin{array}{l}
\mathrm{x}(\mathrm{t})<\begin{array}{c}
\ddot{\mathrm{x}}(\mathrm{t})+\delta \dot{\mathrm{x}}(\mathrm{t})+\beta \mathrm{x}(\mathrm{t})+\alpha\{\mathrm{x}(\mathrm{t})\}^{3} \\
=\gamma \cos (\omega \mathrm{t})+\mathrm{EEG}
\end{array} \\
\dot{\mathrm{x}}(\mathrm{t})<
\end{array}\right\} \gamma \cos (\omega \mathrm{t})+\mathrm{EEG}
$$


One set of sample EEG signals for five distinct emotive stimuli is given in Figure 3. The method of selection of stimulus responsible for arousal of a specific emotion is given in the next section. Once a stimulus responsible for arousal of joy is submitted to the Duffing oscillator, the phase trajectory is constructed using the time-response and its first time derivative at discrete time data points. Figure 4 shows the EEG obtained for a stimulus of joy, the response of the Duffing oscillator to this signal and its corresponding phase-portraits. It needs mention here that phase-portraits depend on the choice of initial values of the oscillator parameters. In Figure 4, we considered $x(0)=0, \dot{x}(0)=0$. It is apparent from the phase trajectory [Figure 4(b)] that the phase portrait has two primary lobes with several overlaps, indicating a clear chaotic behaviour of oscillator dynamics. The nature of the chaos, however, may be different for different audio-visual stimuli.

Figure 3 Time varying plot for the: (a) emotion of anger (b) emotion of fear (c) emotion of joy (d) emotion of relaxation (e) emotion of sadness

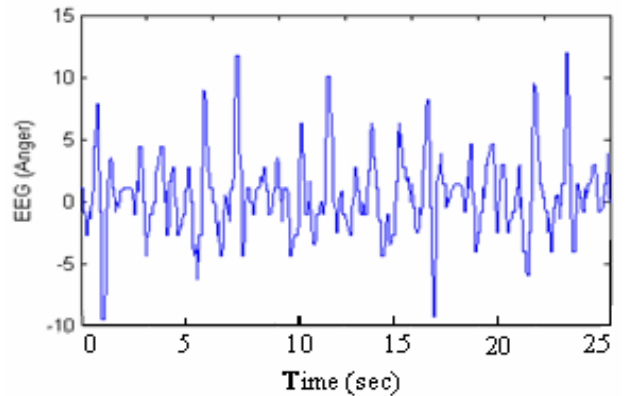

(a)

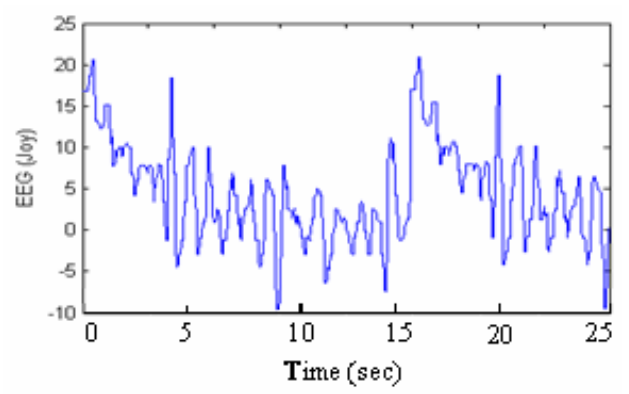

(c)

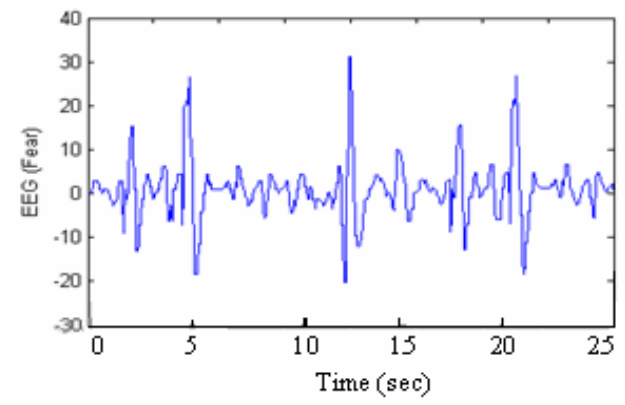

(b)

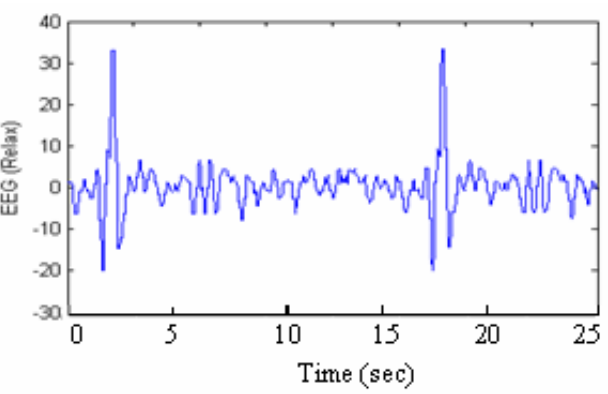

(d)

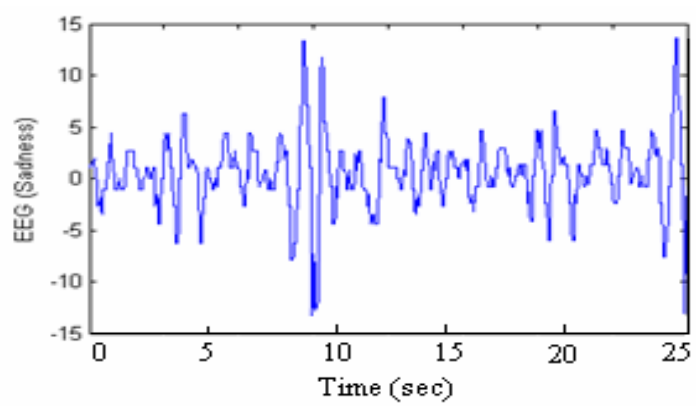

(e) 
Figure 4 (a) Plot for EEG data of joy against time (b) plot for state variable $x$ for EEG data of joy against time (c) plot for state variable $\dot{x}$ for EEG data of joy against time (d) phase trajectory of joy with initial condition at $x(0)=0$ and $\dot{x}(0)=0$

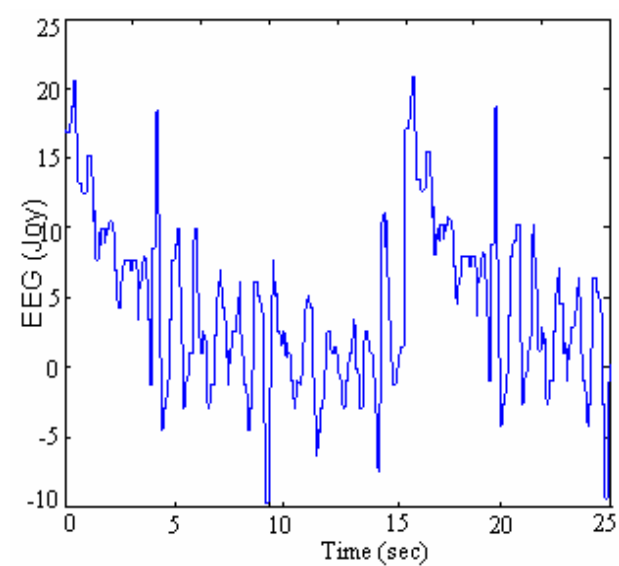

(a)

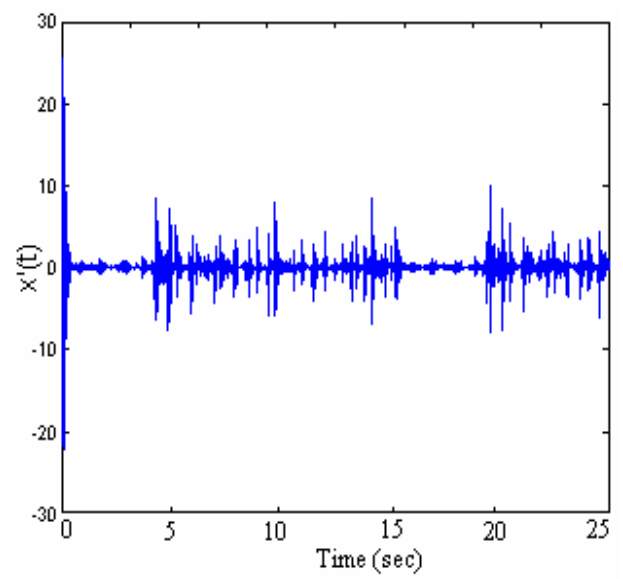

(c)

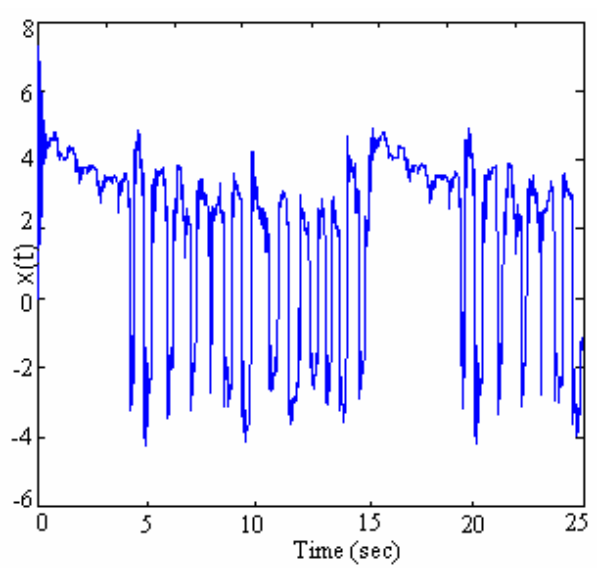

(b)

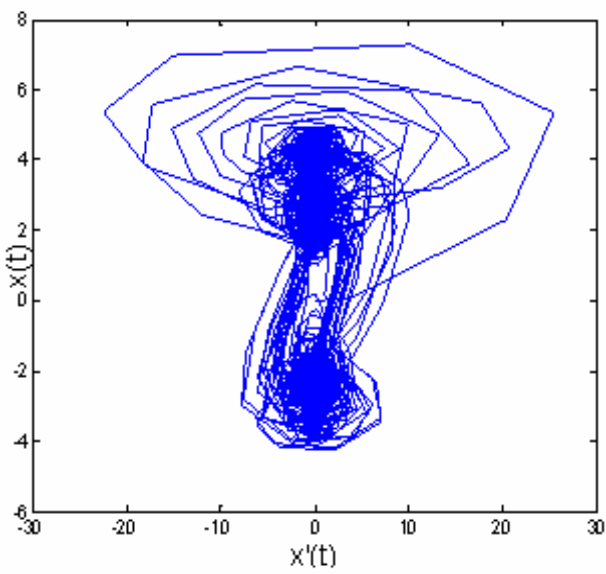

(d)

A variation in the initial condition is studied in Figure 5. It is noted from this figure that the phase trajectory maintains structural similarity with two lobes irrespective of the initial conditions, i.e., $x(0)=0$ and $\dot{x}(0)=0$. In other words, the shape of the phase trajectory for joy is unique with two lobes as obtained in Figures 5(a)-5(c). 
Figure 5 (a) Phase trajectory of emotion joy with initial condition at $x(0)=2$ and $\dot{x}(0)=20$

(b) phase trajectory of emotion joy with initial condition $x(0)=-2$ and $\dot{x}(0)=20$

(c) phase trajectory of emotion joy with initial condition at $x(0)=-2$ and $\dot{x}(0)=-20$

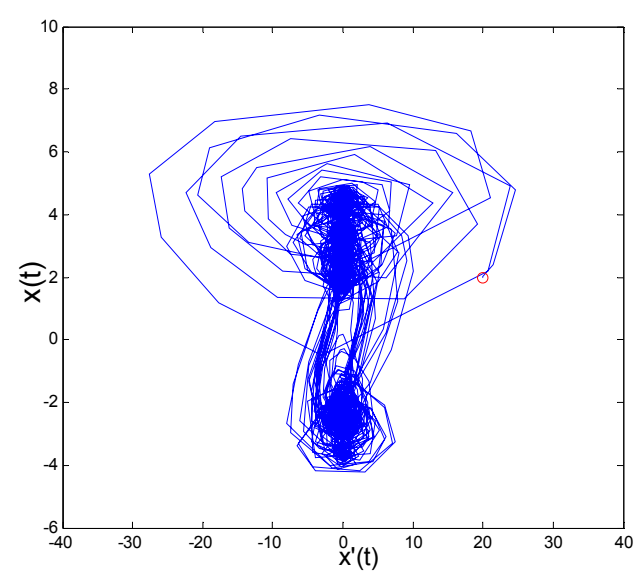

(a)

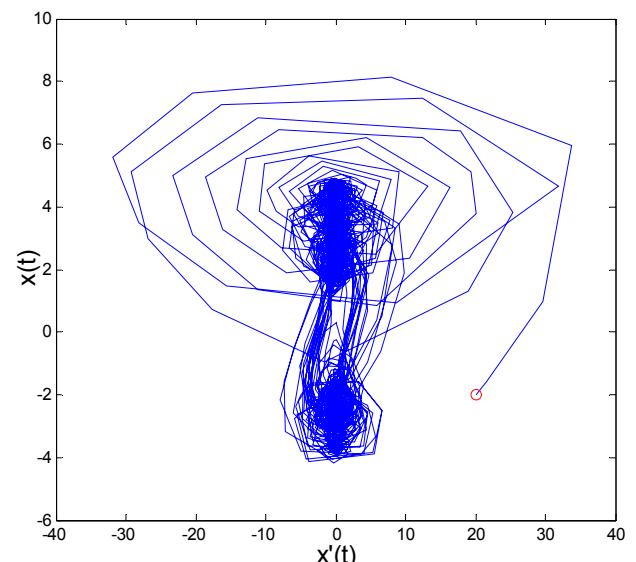

(b)

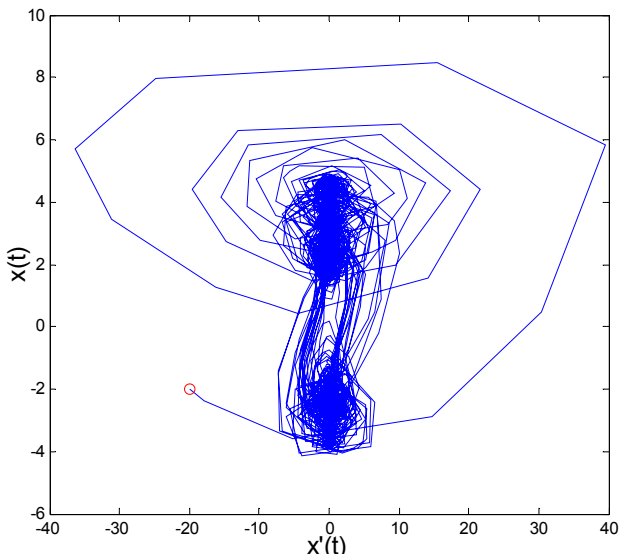

(c) 


\section{Experiments}

The experiment includes three issues. First, we present a scheme for the selection of audio-visual stimulus responsible for excitation of specific emotions of a given subject. Next, we determine the shape of the phase portraits from the time-response of the Duffing oscillator, when excited with a given emotive stimulus. Lastly, we confirm that for similar emotive stimulus the phase portraits also look similar.

The continuous time EEG signal obtained from the first order hold circuit is used to excite the Duffing oscillator (Holmes, 1979; Holmes and Rand, 1976, 1980; Holmes and Holmes, 1981; Holmes and Whitley, 1983; Novak and Frehlich, 1982; Ott, 2002; Srebro, 1995; Thompson and Stewart, 2002; Ueda, 1979, 1980, 1992; Yuan and Li, 2008). The response of the oscillator is obtained by solving the differential equation governing the oscillator dynamics using fourth order numerical integration by the Runge-Kutta method. The experiment was dome using 15 audio-visual stimuli. Each three correspond to exciting a specific emotion. The principle of automatically identifying the best audio-visual stimulus for the excitation of a given emotion is outlined below.

Table 1 Assessment of the arousal potential of selected audio-visual movie clips in exciting different emotions

\begin{tabular}{lcccccc}
\hline $\begin{array}{l}\text { Subjects used to access } \\
\text { the emotion aroused by } \\
\text { the audio-visual clips }\end{array}$ & $\begin{array}{c}\text { Title of } \\
\text { audio-visual } \\
\text { clips }\end{array}$ & \multicolumn{5}{c}{ Percentage arousals of different emotions by a clip } \\
\cline { 3 - 7 } & Clip 1 & 0 & 20 & 80 & 0 & 0 \\
\hline Subject 1 & Clip 1 & 0 & 25 & 75 & 0 & 0 \\
Subject 2 & & & & & & \\
$\ldots$ & Clip 1 & 0 & 12 & 88 & 0 & 0 \\
Subject 50 & Clip 2 & 0 & 82 & 0 & 9 & 9 \\
Subject 1 & Clip 2 & 0 & 80 & 0 & 12 & 8 \\
Subject 2 & & & & & & \\
$\ldots$ & Clip 2 & 0 & 84 & 0 & 10 & 6 \\
Subject 50 & & & & & & \\
$\ldots$ & Clip 60 & 78 & 10 & 0 & 0 & 12 \\
Subject 1 & Clip 60 & 80 & 16 & 0 & 0 & 4 \\
Subject 2 & Clip 60 & 84 & 8 & 0 & 0 & 8 \\
Subject 50 & & & & &
\end{tabular}

\subsection{Stimulus selection}

To identify the correct audio-visual stimulus responsible to arouse a given emotion, the stimuli are initially classified manually. This is done with the help of 50 observers, most of whom are students or the faculty of Jadavpur University. Each observer is asked to classify a given audio-visual stimulus into five emotion-arousing classes, including anger, fear, joy, relaxation and sadness. A 100-point scale is used and individual scores are allotted to the possibility space of the five emotions. The sum of the scores assigned to a given audio-visual stimulus is 100 . For the 50 observers, we determine the mean and variance of their assignments to a particular category. Ultimately the ratio of mean/variance for each of the five emotions is evaluated. The category having the largest mean/variance ratio is considered to be the best appropriate emotion for any given 
stimulus. The experiment is repeated for 60 such stimuli. The mean/variance ratio of the winning emotion for each stimulus is identified. A sorting algorithm is then used to rank the stimuli according to the descending order of their mean/variance measure for the specific emotion category. The first three stimuli for each category of emotion are then identified from this list. The complete experiment is done with the three stimuli responsible for the excitation of a specific emotion. For five emotions, we thus have $5 \times 3=15$ best audio-visual samples.

Table 1 gives the tabular representation of the results obtained by responses obtained from 50 subjects. Each subject is shown 60 sets of audio-visual stimuli. From the table, it is clear that the row-sum give in Table 1 has always a value of 100 .

\subsection{Clustering from the Shape of the phase portraits}

The experiment starts with five emotive stimuli, each responsible for excitation of five distinct emotions: anger, joy, fear, sadness and relaxation. The EEG signals obtained for individual stimulus is recorded and processed, and submitted to the input of a Duffing oscillator. The response to this stimulus is obtained by numerically integrating the dynamics of the oscillator using Runge-Kutta method. The initial conditions of the numerical integration were set to $x=0, y=0$. Phase-trajectories obtained from the oscillator response are plotted for all the five distinct stimulus mentioned above (Table 2). It is noted from Table 2 that individuality of the portraits is maintained for the different excitatory stimuli. The remarks column under Table 2 clearly indicates the special features of the individual phase portraits. Consequently, the EEG signal corresponding to a specific emotion excitatory stimulus can be segregated in the phase space of the oscillator. Here lies the significance of the Duffing oscillator.

The experiment was repeated for 50 different subjects and the results are appealing. It was observed that the phase portrait obtained for most of the subjects for a given stimulus look similar in shape and number of lobes. The misclassification of emotion from the shape-based phase portraits is as low as $6.8 \%$. A comparison with existing literature reveals that the proposed scheme outperforms the traditional schemes of emotion classification from EEG data (Murugappan et al., 2008; Saiwaki et al., 1998).

Table 2 Experimental results on the cluster-shapes

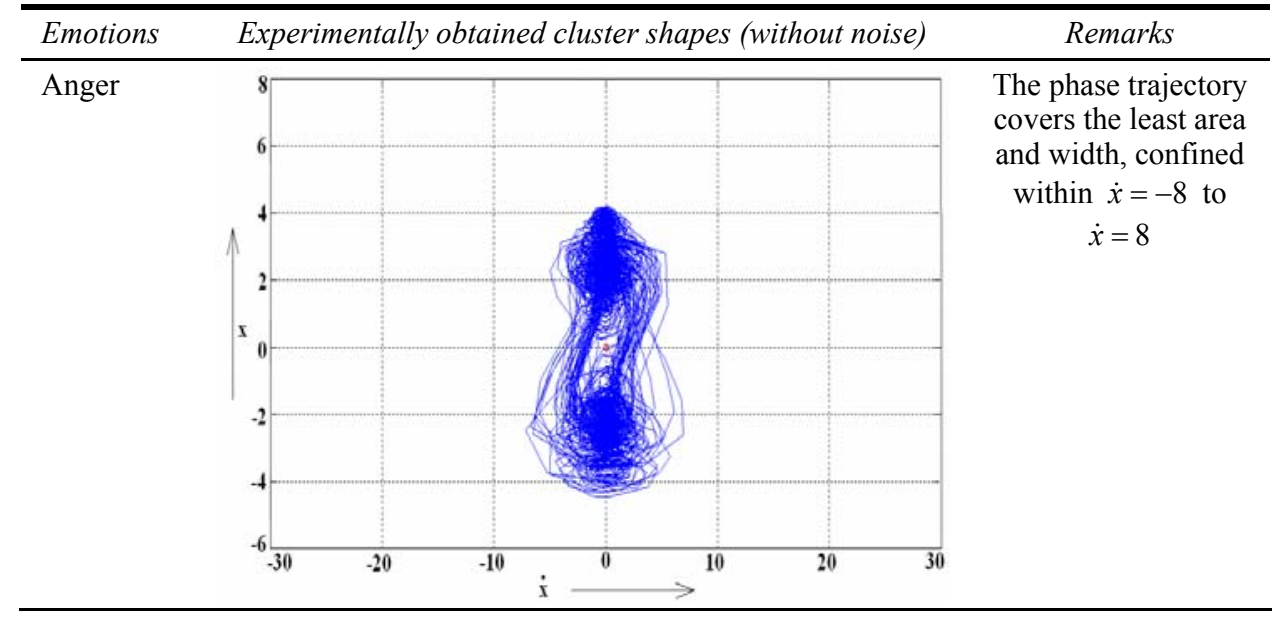


Table 2 Experimental results on the cluster-shapes (continued)

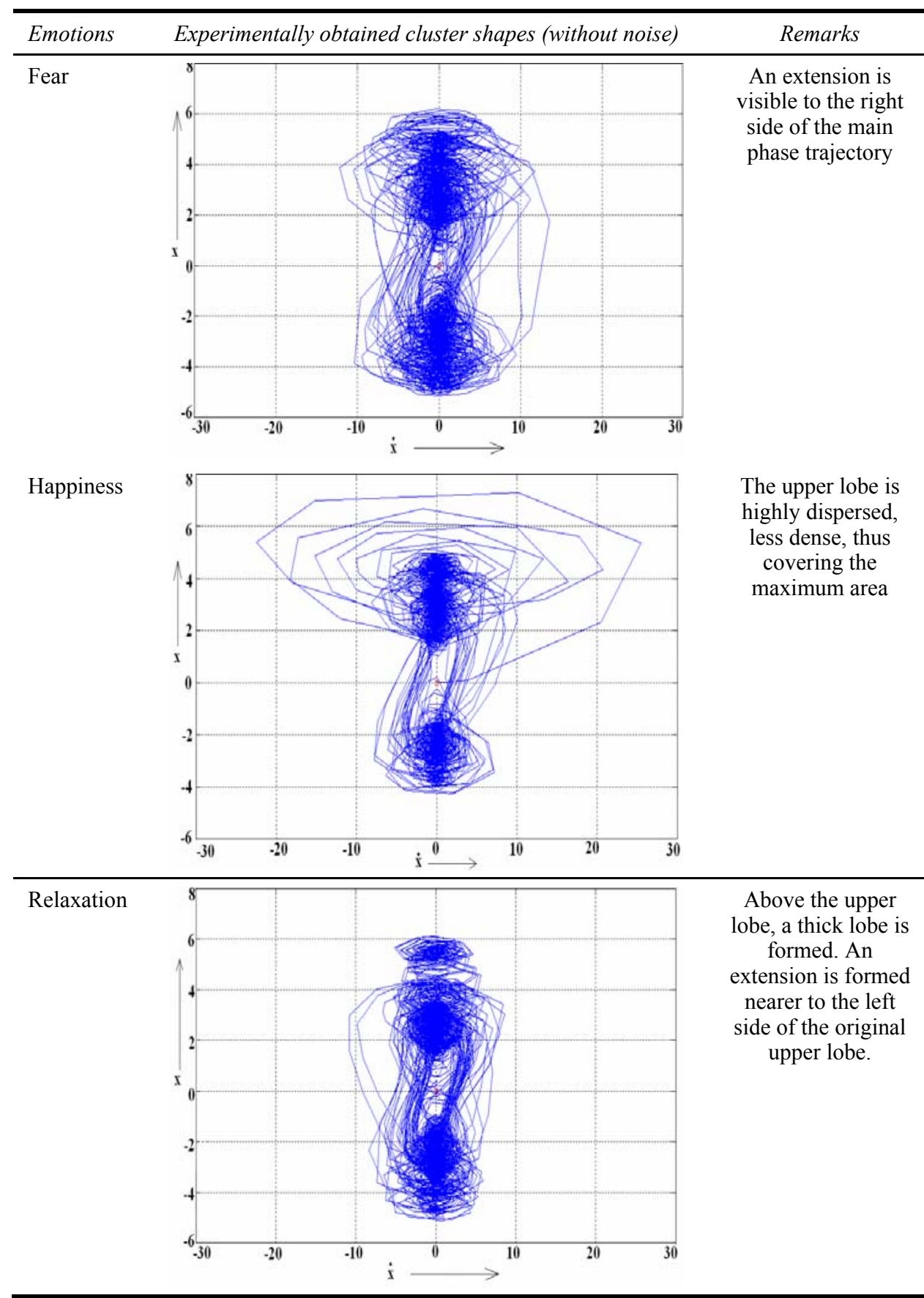


Table 2 Experimental results on the cluster-shapes (continued)

Sadness

Figure 6 Phase trajectory for joy due to: (a) first stimulus of joy (b) second stimulus of joy (c) third stimulus of joy

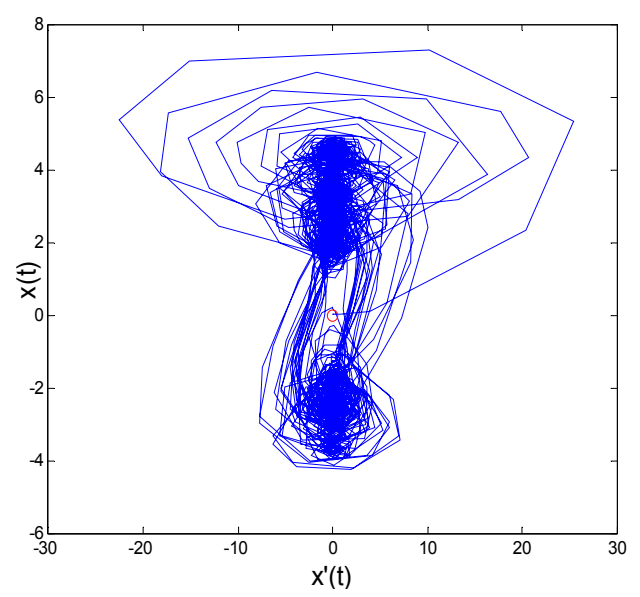

(a)

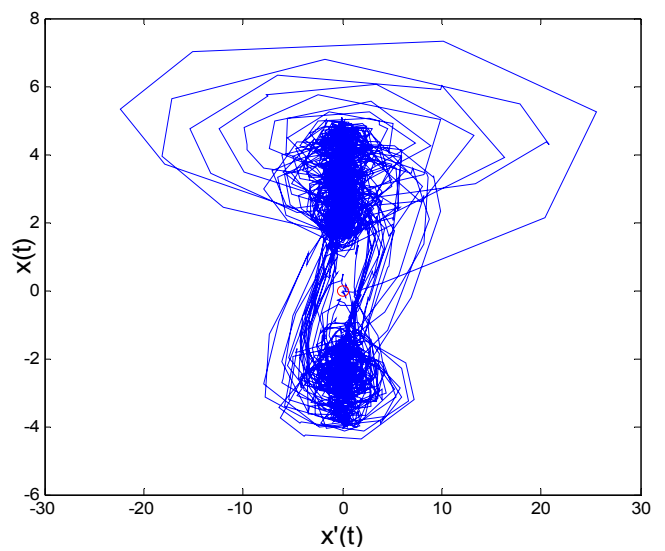

(b) 
Figure 6 Phase trajectory for joy due to: (a) first stimulus of joy (b) second stimulus of joy (c) third stimulus of joy (continued)

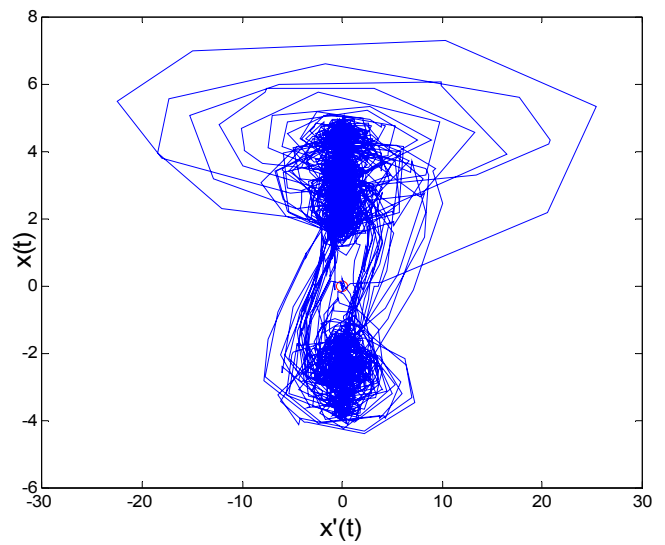

(c)

\subsection{Effect of similar stimuli on clustering}

The objective of this experiment is to study the effect of two or more stimuli causing same emotion on the phase portraits. It may be noted that we already identified three top ranking stimuli responsible to arouse same emotion. These stimuli were applied to the input of the Duffing oscillator, and its corresponding phase-portraits are plotted for joy (Figure 6) and anger (Figure 7). It is apparent from Figure 6 that there exists similarity in phase portraits (a)-(d) obtained for the emotion joy with different stimulus.

To validate the results, the experiment was repeated for the emotion anger and the results too are promising. The phase portraits of anger for three different stimuli are obtained and plotted in Figure 7. It is clear from Figure 7 that the portraits look very similar, signifying the common belief that similar excitations cause similar emotions.

Figure 7 Phase trajectory for anger due to: (a) first stimulus of anger (b) second stimulus of anger (c) third stimulus of anger

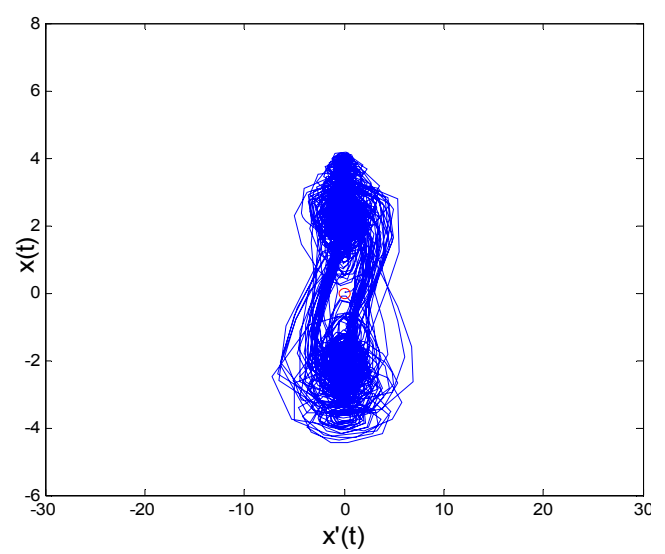

(a) 
Figure 7 Phase trajectory for anger due to: (a) first stimulus of anger (b) second stimulus of anger (c) third stimulus of anger (continued)

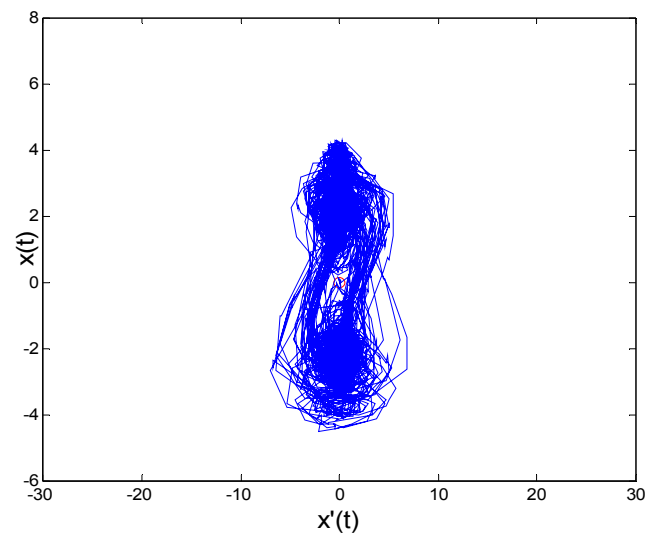

(b)

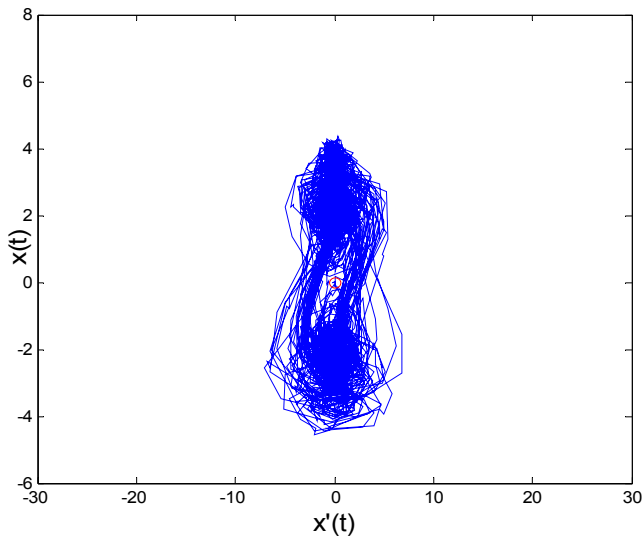

(c)

\section{Noisy clustering}

In this section, we experiment by adding additive white Gaussian noise (AWGN) to the original signal for a specific emotion. We note the changes in the phase portrait obtained from the Duffing oscillator response. The AWGN channel model is one in which the only impairment is the linear addition of wideband or white noise with a constant spectral density. This is expressed as Watts per Hertz of bandwidth and a Gaussian distribution of amplitude. It is noted that when the signal-to-noise ratio (SNR or S/N) of the EEG signal is maintained at a level of $25 \mathrm{~dB}$, the phase portraits maintain similarity, which indicates robustness in emotion clustering. SNR is defined as the ratio of a signal power to the noise power which corrupts the signal. A ratio higher than 1:1 indicates there is more signal than noise. In less technical terms, the SNR compares the level of the desired signal, on here, the obtained EEG signals, to the level of added unwanted noise. The 
higher the ratio, the less obtrusive is the background noise. The value of SNR is given by the following equation:

$$
\operatorname{SNR}(d B)=10 \log _{10}\left(\frac{P_{\text {signal }}}{P_{\text {noise }}}\right)=20 \log _{10}\left(\frac{A_{\text {signal }}}{A_{\text {noise }}}\right)=P_{\text {signal }, d B}-P_{\text {noise }, d B}
$$

Figures 8(a), 8(b), 8(c), 9(a), 9(b), 9(c), 10(a), 10(b) and 10(c) demonstrate the behaviour in the phase portrait for different level of SNR as indicated in the caption. It is noted that when the SNR is less than a certain threshold, misclassification occurs. Note the differences in the phase portraits for a given emotion.

Figure 8 Phase trajectories for fear when the EEG signal is corrupted with noise of an SNR of (a) $30 \mathrm{~dB}$ (b) $25 \mathrm{~dB}$ (c) $20 \mathrm{~dB}$

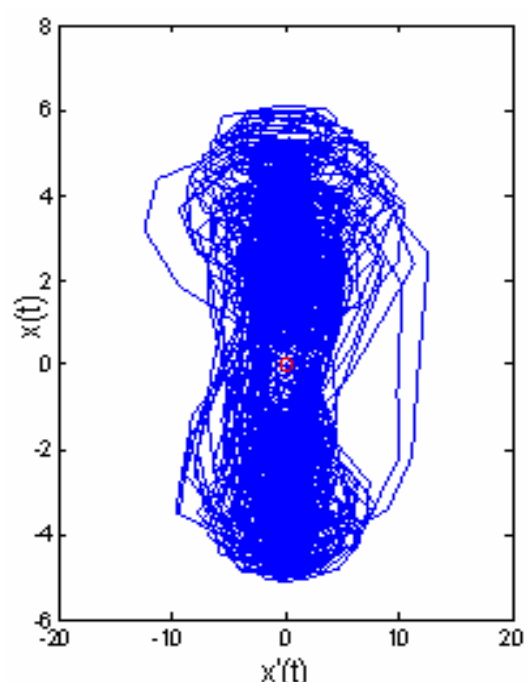

(a)

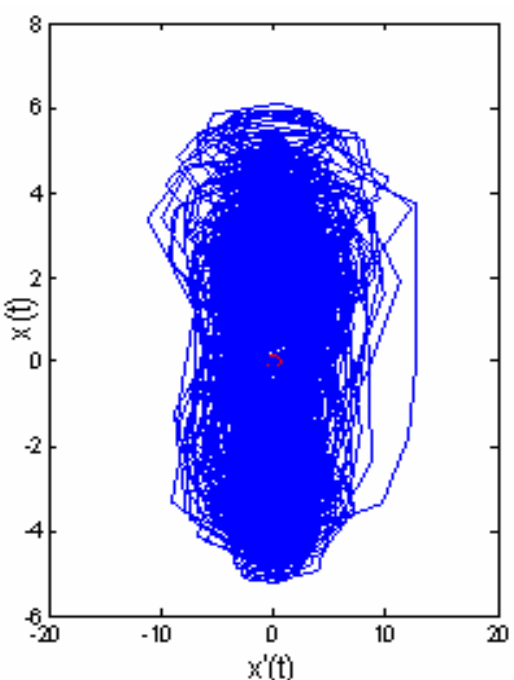

(b)

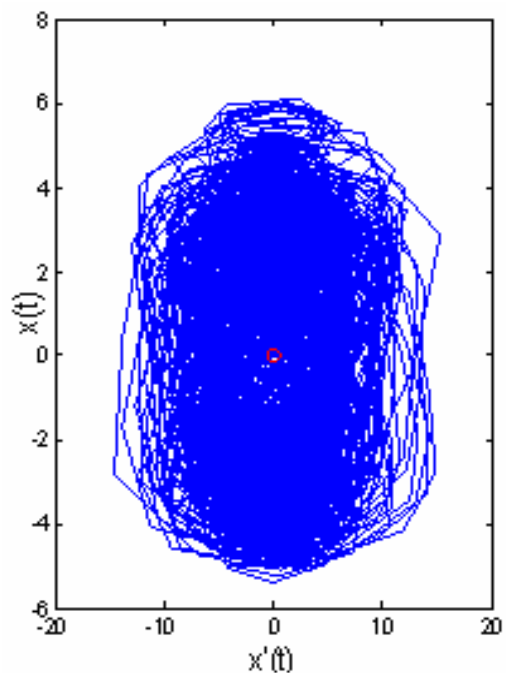

(c) 
Classifications of the phase trajectories of different emotions were based on several features of the phase portrait. These features included:

1 The presence of any third lobe. The values were used as follows; a value of +1 is taken for an extra upper lobe, a -1 is taken for an extra lower lobe, and a 0 is considered for the absence of any extra lobe.

2 The position of the centroid of the extra lobe [coordinates of the centroid are taken to be $(0,0)$ if there is no extra lobe]

3 The maximum and the minimum values of $x$ and $d x / d t$.

Figure 9 Phase trajectories for relaxation when the EEG signal is corrupted with noise of an SNR of (a) $30 \mathrm{~dB}$ (b) $25 \mathrm{~dB}$ (c) $20 \mathrm{~dB}$

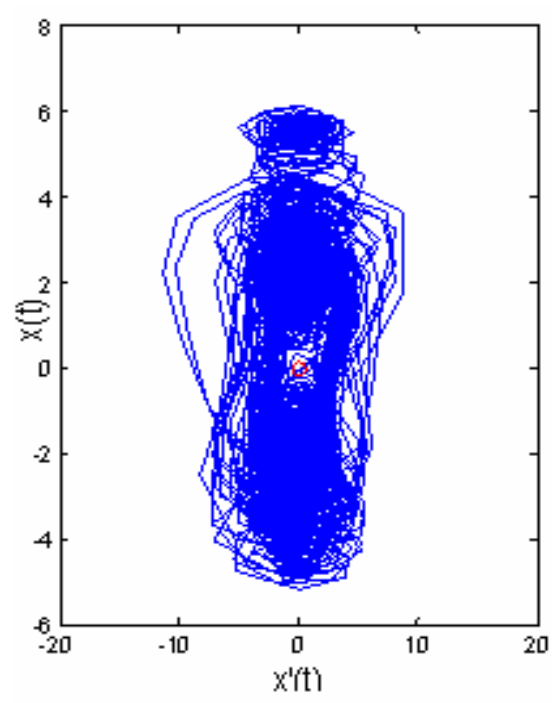

(a)

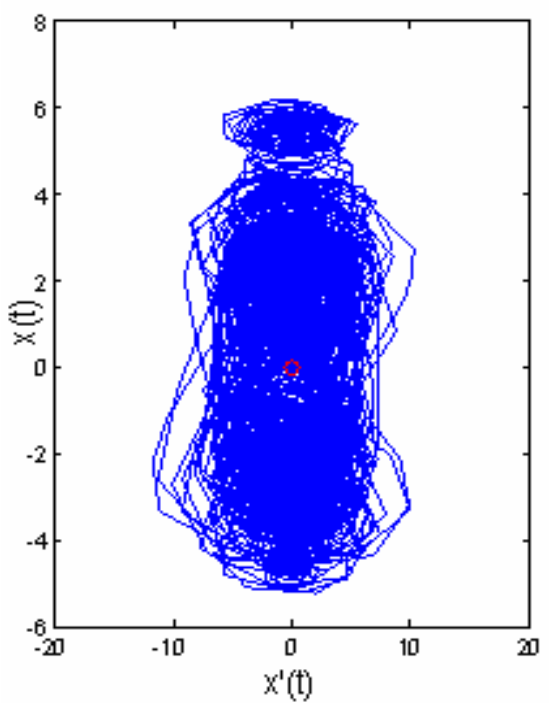

(b)

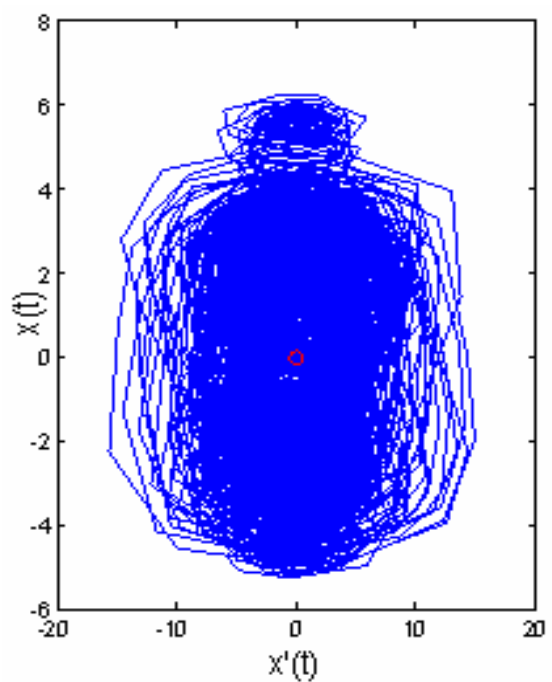

(c) 
Figure 10 Phase trajectories for sadness when the EEG signal is corrupted with noise of an SNR of (a) $30 \mathrm{~dB}$ (b) $25 \mathrm{~dB}$ (c) $20 \mathrm{~dB}$

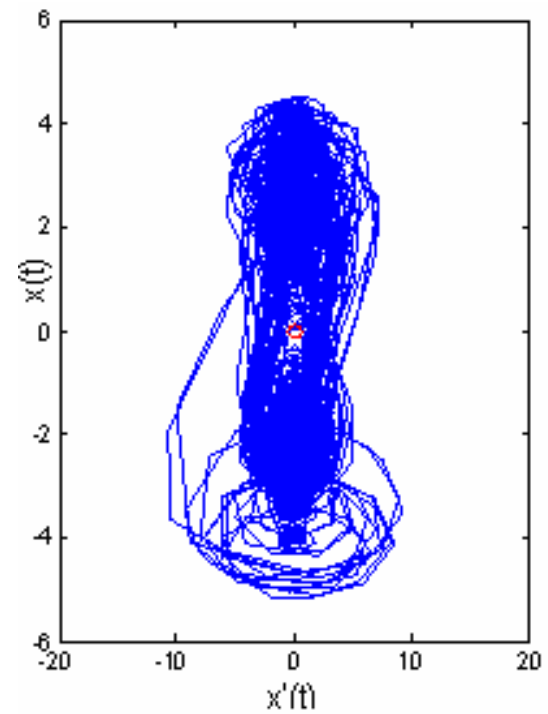

(a)

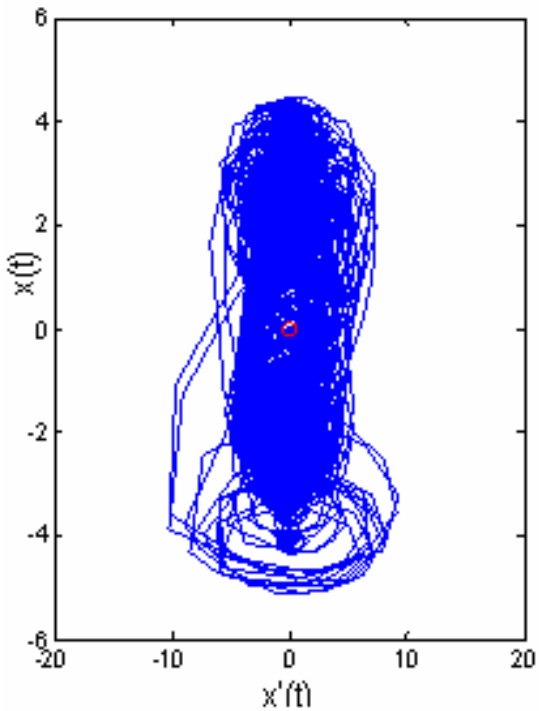

(b)

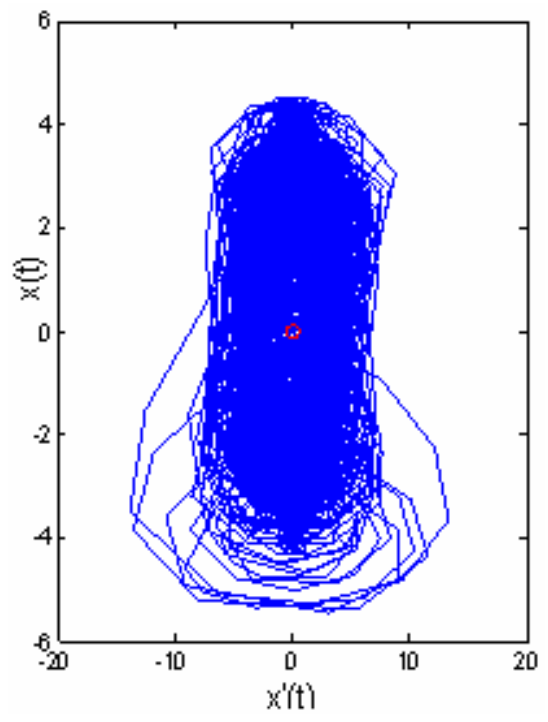

(c)

The features extracted from the phase portraits without noise were then used to train the support vector machine (SVM). The features of the phase portraits obtained from noisy EEG data were classified using the linear one-to-one SVM classifier. SVMs are a set of related supervised learning methods which are used for classification and regression. A SVM constructs a hyperplane or a set of hyperplanes in a high or infinite dimensional space. This then can be used for classification, regression or other tasks. Intuitively, a 
good separation is achieved by a hyperplane that has the largest distance to the nearest training data points of any class. That is the so-called functional margin. In general, larger the margin, lower the generalisation error of the classifier. The one-to-one approach of classification is done by a max-wins voting strategy. Here, every classifier assigns an instance to one of the two classes. The vote for the assigned class is then increased by one vote. Finally, the class with most votes determines the instance classification.

The accuracy of the classification for different SNRs is shown in Table 3. Figure 11 gives a pictorial representation of the same.

It can be seen from Table 3 that misclassification begins at $25 \mathrm{~dB}$. The misclassification percentage is $0.3 \%$ at $\mathrm{SNR}=25 \mathrm{~dB}$.

Table 3 Percentage of classification accuracy at decreasing values of SNR

\begin{tabular}{lc}
\hline SNRs & Accuracy \\
\hline $55 \mathrm{~dB}$ & $100 \%$ \\
$50 \mathrm{~dB}$ & $100 \%$ \\
$45 \mathrm{~dB}$ & $100 \%$ \\
$40 \mathrm{~dB}$ & $100 \%$ \\
$35 \mathrm{~dB}$ & $100 \%$ \\
$30 \mathrm{~dB}$ & $100 \%$ \\
$25 \mathrm{~dB}$ & $99.7 \%$ \\
$20 \mathrm{~dB}$ & $98.3 \%$ \\
$15 \mathrm{~dB}$ & $92.6 \%$ \\
$10 \mathrm{~dB}$ & $84.4 \%$ \\
\hline
\end{tabular}

Figure 11 Variation of classification accuracy with various amount of noise (see online version for colours)

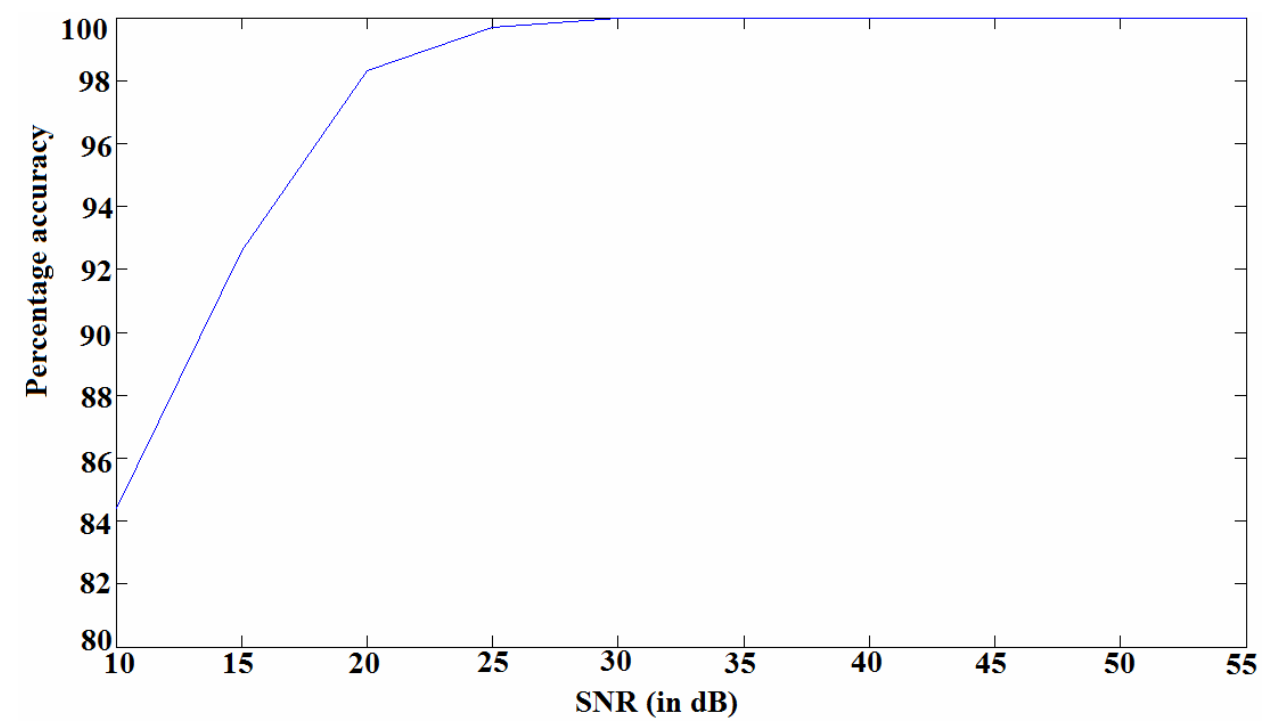




\section{Conclusions}

The paper employed Duffing oscillator for emotion clustering from stimulated EEG signals. Experiments with 50 subjects confirmed that the mapping of EEG to phase portraits of Duffing oscillator for a given stimulus is unique. This justifies high classification accuracy of emotion from stimulated EEG samples by the proposed approach. Experiments further reveal that similar stimulus responsible for arousal of a specific emotion yields similar phase portraits, signifying similarity in the EEG samples for the stimuli. Analysis with noisy EEG data demonstrates that for SNR up to $25 \mathrm{~dB}$, the emotion clustering can be performed from the EEG samples without error. Above all, the paper envisages that the EEG signal itself is more important for emotion clustering and the performance of unsupervised clustering by Duffing oscillator is better in classification accuracy than other EEG-based supervised classification and other modes of emotion classification.

\section{References}

Bos, D. (2007) 'EEG-based emotion recognition', available at http://hmi.ewi.utwente.nl/verslagen/capita-selecta/CS-Oude Bos-Danny.pdf.

Carroll, T.L. and Pecora, L.M. (1993) 'Stochastic resonance and chaos', Phys. Rev. Lett., Vol. 70, pp.576-579.

Chakroborty, A. (2005) 'Cognitive cybernetics - a study of the behavioral models of human, interactions', $\mathrm{PhD}$ thesis, Jadavpur University.

Chakroborty, A. and Konar, A. (2009) Emotional Intelligence: A Cybernetic Approach, Springer.

Ditto, W.L., Rauseo, S.N. and Spano, M.L. (1990) 'Experimental control of chaos', Phys. Rev. Lett., Vol. 26, pp.3211-3214.

Dolan, R.J., Heinze, H.J., Hurlemann, R. and Hinrichs, H. (2006) 'Magnetoencephalography (MEG) determined temporal modulation of visual and auditory sensory processing in the context of classical conditioning to faces', Neuroimage, Vol. 32, No. 2, pp.778-789.

Ekman, P. and Friesen, W.V. (1975) Unmasking the Face: A Guide to Recognizing Emotions from Facial Clues, Prentice-Hall, Englewood Cliffs, NJ.

Holmes, C. and Holmes, P. (1981) 'Second order averaging and bifurcations to subharmonics in Duffing's equation', Journal of Sound and Vibration, Vol. 78, pp.161-174.

Holmes, P. (1979) 'A nonlinear oscillator with a strange attractor', Philosophical Transactions of the Royal Society A, Vol. 292, pp.419-448.

Holmes, P. and Rand, D. (1980) 'Phase portraits and bifurcations of the non-linear oscillator', International Journal of Non-linear Mechanics, Vol. 15, pp.449-458.

Holmes, P. and Whitley, D. (1983) 'On the attracting set for Duffing's equation, II. A geometrical model for moderate force and damping', Physica, Vol. 7D, pp.111-123.

Holmes, P.J. and Rand, D.A. (1976) 'The bifurcations of Duffing's equation: an application of catastrophe theory', Journal of Sound and Vibration, Vol. 44, pp.237-253.

Johnstone, T., van Reekum1, C.M., Oakes, T.R. and Davidson, R.J. (2006) 'The voice of emotion: an FMRI study of neural responses to angry and happy vocal expressions', Social Cognitive and Affective Neuroscience, 20 October, Vol. 1, No. 3, pp.242-249.

Khalili, Z. and Moradi, M.H. (2008) 'Emotion detection using brain and peripheral signals', Proceedings of the IEEE Cairo Int. Biomedical Engineering Conf. (CIBEC'08), 18-20 December. 
Khalili, Z. and Moradi, M.H. (2009) 'Emotion recognition system using brain and peripheral signals: using correlation dimension to improve the results of EEG', Proceedings of International Joint Conference on Neural Networks, Atlanta, Georgia, USA, 14-19 June.

Konar, A. and Jain, L.C. (2005) Cognitive Engineering, Springer.

Kuo, B.C. (2002) Automatic Control Systems, 8th ed., John Wiley \& Sons Inc.

Li, M., Chai, Q., Kaixiang, T., Wahab, A. and Abut, H. (2009) 'EEG emotion recognition system', Book Chapter, In-Vehicle Corpus and Signal Processing for Driver Behavior, pp.1-11, Springer, USA.

Murugappan, M., Rizon, M., Nagarajan, R. and Yaacob, S. (2008) 'EEG feature extraction for classifying emotions using FCM and FKM', Proceedings of the 7th WSEAS International Conference on Applied Computer and Applied Computational Science, Hangzhou, China, pp.299-304, ISBN ISSN:1790-5117, 978-960-6766-61-9.

Novak, S. and Frehlich, R.G. (1982) 'Transition to chaos in the Duffing oscillator', Physical Review A, APS.

Ogata, K. (2009) Modern Control Engineering, 5th ed., 4 September, Prentice Hall.

Ott, E. (2002) Chaos in Dynamical Systems, 2nd ed., Cambridge University Press.

Peyk, P., Schupp, H.T., Elbert, T. and Junghöfer, M. (2008) 'Emotion processing in the visual brain: a MEG analysis', Brain Topography, June, Vol. 20, No. 4, pp.205-215, Springer, New York.

Russell, J.A., Bachorowski, J-A. and Fernández-Dols, J-M.(2003) 'Facial and vocal expressions of emotion', Vol. 54, pp.329-349, (volume publication date February 2003) doi:10.1146/annurev.psych.54.101601.145102, first published online as a review in advance on 4 October 2002.

Saiwaki, N., Kimura, N. and Nishida, S. (1998) 'An analysis of EEGS based on information flow with SD method', IEEE Int. Conf. on Systems, Man and Cybernetics, 11-14 October.

Schneider, F., Grodd, W., Gur, R.E., Klose, U., Alavi, A. and Gur, R.C. (1997) 'PET and fMRI in the study of emotion', Psychiatry Research: Neuroimaging, 7 February, Vol. 68, Nos. 2-3, pp.174-175.

Srebro, R. (1995) 'The Duffing oscillator: a model for the dynamics of the neuronal groups comprising the transient evoked potential', Electroencephalography and Clinical Neurophysiology/Evoked Potentials Section, November, Vol. 6, No. 6, pp.561-573, Elsevier Science Ireland Ltd.

Takahashi, K. (2004) 'Remarks on emotion recognition from bio-potential signals', 2nd International Conference on Autonomous Robots and Agents, 13-15 December, Palmerston North, New Zealand.

Thompson, J.M.T. and Stewart, H.B. (2002) Nonlinear Dynamics and Chaos, 2nd ed., John Wiley $\&$ Sons.

Ueda, Y. (1979) 'Randomly transitional phenomena in the system governed by Duffing's equation', Journal of Statistical Physics, Vol. 20, pp.181-196.

Ueda, Y. (1980) 'Explosion of strange attractors exhibited by Duffing's equation', in R.H.G. Helleman (Ed.): Nonlinear Dynamics, pp.422-434, New York Academy of Science.

Ueda, Y. (1992) The Road to Chaos, Aerial Press.

Yoshitomi, Y., Kim, S-I., Kawano, T. and Kilazoe, T. (2000) 'Effect of sensor fusion for recognition of emotional states using voice, face image and thermal image of face', Robot and Human Interactive Communication, RO-MAN 2000. Proceedings 9th IEEE International Workshop on, 27-29 September, pp.178-218.

Yuan, Y. and Li, Y. (2008) 'Study on EEG time series based on Duffing equation', BMEI, 2008 International Conference on BioMedical Engineering and Informatics, Vol. 2, pp.516-519. 